\title{
Generally Covariant Duality and Tachyon of Cosmological Nature
}

\author{
Dao Vong Duc ${ }^{1}$, Phu Chi Hoa ${ }^{2}$ \\ ${ }^{1}$ Institute of Physics, Hanoi, Vietnam \\ ${ }^{2}$ Dalat University, Dalat, Vietnam \\ Email: dvduc@iop.vast.ac.vn, hoapc@dlu.edu.vn
}

Received 13 October 2014; revised 12 November 2014; accepted 5 December 2014

Copyright (C) 2014 by authors and Scientific Research Publishing Inc.

This work is licensed under the Creative Commons Attribution International License (CC BY). http://creativecommons.org/licenses/by/4.0/

c) (i) Open Access

\begin{abstract}
In this work the concept of generally covariant duality is treated with the introduction of generalized Levi-Civita tensor within the framework of vierbein formalism. The equations for the attached Lorentz scalar fields are derived from vierbein postulate. It is shown that the masses of the associated particles including that with negative square mass are completely determined by Einstein's cosmological constant.
\end{abstract}

\section{Keywords}

General Relativity, Cosmology, Tachyon

\section{Motivation}

Recently there have been many attempts to approach the problem of unification of fundamental interactions on the base of Extended General Relativity [1]. The starting insight is that all the fundamental interactions are originated from the nature of space-time itself as the gravitational interaction is.

On the other side, superstring theory [2] [3] is also considered as a promising approach to the construction of the unification theory. Among the most notable theoretical consequences of string theory is the existence of tachyon-scalar particle having negative square mass. In this connection we would mention that according to the results obtained in Refs. [4]-[6] the existence of tachyons could be originated from the compactification of timelike extradimensions.

The aim of this work is to consider the concept of generally covariant duality in General Relativity with the introduction of generalized Levi-Civita tensor and to study the specific properties of the attached fields within the framework of vierbein formalism.

It is shown that the masses of the associated particles, in particular tachyon-like particle, are completely de- 
termined by Einstein’s cosmological constant.

\section{Generally Covariant Duality}

In special Relativity the Duality concept is treated by means of the 4-rank Levi-Civita tensor $\varepsilon_{\mu \nu \lambda \rho}$. The well known example is the relation

$$
\tilde{F}_{\mu \nu}=\frac{1}{2} \varepsilon_{\mu \nu \lambda \rho} F^{\lambda \rho}
$$

for electromagnetic field strength tensor

with the identification

$$
F^{\lambda \rho} \equiv \frac{\partial A^{\rho}}{\partial x_{\lambda}}-\frac{\partial A^{\lambda}}{\partial x_{\rho}}
$$

$$
F^{o i}=E^{i}, F^{23}=H^{1}, F^{31}=H^{2}, F^{12}=H^{3}
$$

for electric $\boldsymbol{E}$ and magnetic $\boldsymbol{H}$ fields, Equation (1) represents Maxwell's equations.

General Relativity requires the generalized version of $\varepsilon_{\mu \nu \lambda \rho}$, which is to be some fully antisymmetric 4-rank tensor denoted by $E_{\mu \nu \lambda \rho}(x)$. In this connection the covariant wedge product of two vectors $A_{\mu}$ and $B_{\mu}$ is to be defined as:

$$
(A \wedge B)_{\mu \nu}=E_{\mu \nu \lambda \rho}(x) A^{\lambda} B^{\rho}
$$

and in correspondence the relation (1) is modified to become

$$
\tilde{F}_{\mu \nu}=(D \wedge A)_{\mu \nu} \equiv E_{\mu \nu \lambda \rho}(x) D^{\lambda} A^{\rho}=\frac{1}{2} E_{\mu \nu \lambda \rho}(x) F^{\lambda \rho}
$$

where $D$ denotes covariant derivative,

$$
D_{\lambda} A_{\rho} \equiv \partial_{\lambda} A_{\rho}-\Gamma_{\lambda \rho}^{\sigma} A_{\sigma}
$$

$\Gamma_{\lambda \rho}^{\sigma}$ being affine connection.

Let the tensor $E_{\mu \nu \lambda \rho}(x)$ under consideration have $e_{a b c d}$ as its vierbein component, namely:

$$
\begin{aligned}
& E_{\mu \nu \lambda \rho}(x)=e_{a b c d} \cdot v_{\mu}^{(a)} v_{v}^{(b)} v_{\lambda}^{(c)} v_{\rho}^{(d)}(x) \\
& e_{a b c d}=E_{\mu \nu \lambda \rho}(x) \cdot v_{(a)}^{\mu} v_{(b)}^{v} v_{(c)}^{\lambda} v_{(d)}^{\rho}(x)
\end{aligned}
$$

where $v_{\mu}^{(a)}(x), \cdots$ stands for vierbein, ( $a, b, \cdots$ being vierbein indices) statisfying the relations with metric tensors:

$$
\begin{aligned}
& g^{\mu v}(x) v_{\mu}^{(a)} v_{v}^{(b)}=\eta^{a b} \\
& \eta_{a b} v_{\mu}^{(a)} v_{v}^{(b)}=g_{\mu v}(x)
\end{aligned}
$$

$g_{\mu \nu}$ and $\eta_{a b}$ being Riemann and Minkowski metric.

Together with $E_{\mu \nu \lambda \rho}(x)$ let us also consider its contravariant partner

with the convention

$$
E^{\mu \nu \lambda \rho}(x)=g^{\mu \alpha} g^{\nu \beta} g^{\lambda \gamma} g^{\rho \delta} E_{\alpha \beta \gamma \delta}(x)=e^{a b c d} v_{(a)}^{\mu} v_{(b)}^{v} v_{(c)}^{\lambda} v_{(d)}^{\rho}(x)
$$

$$
e_{0123}=-e^{0123}=1 .
$$

Like for Riemann metric $g_{\mu v}(x)$ which is expressed as

$$
g_{\mu v}(x)=\eta_{\mu v}+\gamma h_{\mu v}(x)
$$

( $\gamma$-gravitational constant, $h_{\mu \nu}$-gravitational field), here we can put:

$$
\begin{aligned}
& E_{\mu \nu \lambda \rho}(x)=e_{\mu \nu \lambda \rho} \cdot B(x) \\
& E^{\mu \nu \lambda \rho}(x)=e^{\mu \nu \lambda \rho} \cdot C(x)
\end{aligned}
$$


where $B(x)$ and $C(x)$ are some one-component fields and $B(x), C(x) \rightarrow 1$ in the limit of flat space-time. Under general transformation.

$$
x^{\mu} \rightarrow x^{\prime \mu}=f^{\mu}(x)
$$

they transform according to the rule:

$$
\begin{aligned}
& B^{\prime}\left(x^{\prime}\right)=J \cdot B(x) \\
& C^{\prime}\left(x^{\prime}\right)=J^{-1} \cdot C(x)
\end{aligned}
$$

$J$ being Jacobian transformation determinant.

$$
J \equiv \operatorname{det}\left(\frac{\partial x^{\mu}}{\partial x^{\prime \nu}}\right) .
$$

The Formula (8) tells that the fields $B(x)$ and $C(x)$ are scalar with respect to Lorentz transformation only, but instead $\frac{B(x)}{\sqrt{g}}$ and $\sqrt{g} C(x)$ are scalar with respect to general transformation, $g \equiv \operatorname{det}\left(g_{\mu v}\right)$. With respect to space inverse transformation, $\boldsymbol{x}^{\prime}=-\boldsymbol{x}$, they both behave like pseudoscalar,

$$
\begin{aligned}
& B^{\prime}\left(x^{0},-x\right)=-B(x) \\
& C^{\prime}\left(x^{0},-x\right)=-C(x) .
\end{aligned}
$$

From Equations (4)-(7) it follows that the fields $B(x)$ and $C(x)$ have the following vierbein structure:

$$
\begin{aligned}
& B(x)=-\frac{1}{4} e^{\mu \nu \lambda \rho} \cdot e_{a b c d} v_{\mu}^{(a)} v_{v}^{(b)} v_{\lambda}^{(c)} v_{\rho}^{(d)} \equiv \operatorname{det}\left(v_{\mu}^{(a)}\right) \\
& C(x)=-\frac{1}{4} e_{\mu \nu \lambda \rho} \cdot e^{a b c d} v_{(a)}^{\mu} v_{(b)}^{v} v_{(c)}^{\lambda} v_{(d)}^{\rho} \equiv \operatorname{det}\left(v_{(a)}^{\mu}\right)
\end{aligned}
$$

where $\left(v_{\mu}^{(a)}\right)$ denotes the matrix having $v_{\mu}^{(a)}$ as element in row a and column $\mu,\left(v_{(a)}^{\mu}\right)$-matrix having $v_{(a)}^{\mu}$ as element in row $\mu$ and column $a$,

$$
v_{(a)}^{\mu} \equiv g^{\mu v} \eta_{a b} v_{v}^{(b)} .
$$

Note also that:

$$
B(x)=C^{-1}(x) .
$$

In this sense $B(x)$ and $C(x)$ might be referred to as dual partners.

\section{Dual Equations}

We now derive the equations for $B(x)$ and $C(x)$, starting from vierbein postulate

$$
\nabla_{\alpha} v_{\mu}^{(a)}(x)=0 .
$$

From the vierbein structure (4) and (6) this gives:

$$
\begin{aligned}
& \nabla_{\alpha} E_{\mu \nu \lambda \rho}(x)=0 \\
& \nabla_{\alpha} E^{\mu \nu \lambda \rho}(x)=0 .
\end{aligned}
$$

By inserting (7) into (13) we have:

$$
\begin{aligned}
& \partial_{\alpha} B(x)+\Gamma_{\alpha \mu}^{\mu} B(x)=0 \\
& \partial_{\alpha} C(x)-\Gamma_{\alpha \mu}^{\mu} C(x)=0 .
\end{aligned}
$$

And hence: 


$$
\begin{aligned}
& \left\{\square-\eta^{\alpha \beta} \Gamma_{\alpha \mu}^{\mu} \Gamma_{\beta \nu}^{v}-\partial^{\alpha} \Gamma_{\alpha \mu}^{\mu}\right\} B(x)=0 \\
& \left\{\square-\eta^{\alpha \beta} \Gamma_{\alpha \mu}^{\mu} \Gamma_{\beta \nu}^{v}-\partial^{\alpha} \Gamma_{\alpha \mu}^{\mu}\right\} C(x)=0 \\
& \left(\square \equiv \eta^{\alpha \beta} \partial_{\alpha} \partial_{\beta} \equiv \partial^{\alpha} \partial_{\alpha}\right) .
\end{aligned}
$$

From the expression of $\Gamma_{\alpha \beta}^{\mu}$ we have:

$$
\Gamma_{\alpha \mu}^{\mu}=\frac{1}{2 g} \cdot \partial_{\alpha} g .
$$

Up to first order in gravitational constant $\gamma$ the calculations give:

$$
\begin{aligned}
& g=-\left(1+\gamma h_{\mu}^{\mu}\right) \\
& \Gamma_{\alpha \mu}^{\mu}=\frac{1}{2 g} \gamma \cdot \partial_{\alpha} h_{\mu}^{\mu}
\end{aligned}
$$

where $h_{\mu}^{\mu} \equiv \eta^{\mu v} \cdot h_{\mu v}$.

Equations (15) with the expressions (17) inserted gives:

$$
\begin{aligned}
& \left\{\square+\frac{1}{2} \gamma \cdot \square h_{\mu}^{\mu}\right\} B(x)=0 \\
& \left\{\square-\frac{1}{2} \gamma \cdot \square h_{\mu}^{\mu}\right\} C(x)=0 .
\end{aligned}
$$

On the other hand, by performing similar calculations for the Ricci tensor we obtain:

$$
\begin{aligned}
& R_{\mu \nu} \approx \frac{1}{2} \gamma \cdot\left(\partial_{\mu} \partial_{v} h_{\sigma}^{\sigma}+\square h_{\mu \nu}-\partial_{\mu} \partial^{\sigma} h_{\sigma v}-\partial_{\nu} \partial^{\sigma} h_{\sigma \mu}\right) \\
& R \approx \gamma\left(\square h_{\mu}^{\mu}-\partial^{\mu} \partial^{v} h_{\mu \nu}\right) .
\end{aligned}
$$

Hence, Equations (18) can be rewritten as:

$$
\begin{aligned}
& \left\{\square+\frac{1}{2} R+\frac{1}{2} \gamma \cdot \partial^{\mu} \partial^{v} h_{\mu v}\right\} B(x)=0 \\
& \left\{\square-\frac{1}{2} R-\frac{1}{2} \gamma \cdot \partial^{\mu} \partial^{v} h_{\mu v}\right\} C(x)=0 .
\end{aligned}
$$

By inserting here the expression of $R$,

$$
R=4 \Lambda+8 \pi \gamma \cdot T_{\mu}^{\mu}
$$

derived from Einstein's equation with cosmological constant $\Lambda$,

$$
\frac{1}{2} g_{\mu v} R-R_{\mu \nu}=8 \pi \gamma \cdot T_{\mu v}+\lambda g_{\mu v}
$$

( $T_{\mu \nu}$ denotes energy-momentum tensor of matter field) we have:

$$
\begin{aligned}
& (\square+2 \Lambda) B(x)=-J(x) \cdot B(x) \\
& (\square-2 \Lambda) C(x)=J(x) \cdot C(x)
\end{aligned}
$$

where

$$
J(x) \equiv \gamma\left(\frac{1}{2} \partial^{\mu} \partial^{v} h_{\mu \nu}+4 \pi T_{\mu}^{\mu}\right) .
$$

Equation (23) tells that the fields $B(x)$ and $C(x)$ have square mass equaling

$$
m_{B}^{2}=-m_{C}^{2}=2 \Lambda .
$$


This corresponds to the following Lagrangian terms describing the fields $B(x)$ and $C(x)$ interacting with the gravitational field:

$$
\begin{aligned}
& \mathcal{L}(B)=\frac{1}{2} \partial^{\mu} B \cdot \partial_{\mu} B+\Lambda B^{2}+\frac{1}{2} j(x) B^{2} \\
& \mathcal{L}(C)=\frac{1}{2} \partial^{\mu} C \cdot \partial_{\mu} C-\Lambda C^{2}-\frac{1}{2} j(x) C^{2} .
\end{aligned}
$$

This also means that one of them is tachyon-like particle unless $\Lambda=0$, when they both are massless.

\section{Conclusion}

In this work we consider the concept of Generally Covariant Duality. The focus point is the generalization of flat Levi-Civita tensor for the case of curved Riemann space-time. This leads to some kind of pseudoscalar fields of cosmological nature with the masses closely related to Einstein's cosmological constant. In particular among them there is tachyon-like particle having negative square mass. Taking into account that the cosmological constant has a close relation to dark energy, one might think about the possibility for tachyon to be among the candidates for dark matter.

\section{References}

[1] del Aguila, F., de Azcarraga, J.A. and Albabez, L.E. (1985) Supersymmetry, Supergravity and Related Topics. World Scientific, Singapore City.

[2] Green, M.B., Schwarz, J.H. and Witten, E. (1987) Superstring Theory. Cambridge University Press, Cambridge.

[3] Furlan, G., Jengo, R., Pati, J.C., Sciama, D.W., Sergin, E. and Shafi, Q. (1997) Superstrings, Supergravity and Unified Theories. World Scientific, Singapore City.

[4] Duc, D.V. and Giao, N.M. (2013) A Mechanism for Mass Creation from Extradimensions. arXiv-hepth/1301-1405.

[5] Duc, D.V. and Giao, N.M. (2013) Journal of Modern Physics, 4, 991. http://dx.doi.org/10.4236/jmp.2013.47133

[6] Duc, D.V., Giao, N.M. and Dung, T.T. (2014) Journal of Physical Science and Applications, in Print. 
Scientific Research Publishing (SCIRP) is one of the largest Open Access journal publishers. It is currently publishing more than 200 open access, online, peer-reviewed journals covering a wide range of academic disciplines. SCIRP serves the worldwide academic communities and contributes to the progress and application of science with its publication.

Other selected journals from SCIRP are listed as below. Submit your manuscript to us via either submit@scirp.org or Online Submission Portal.
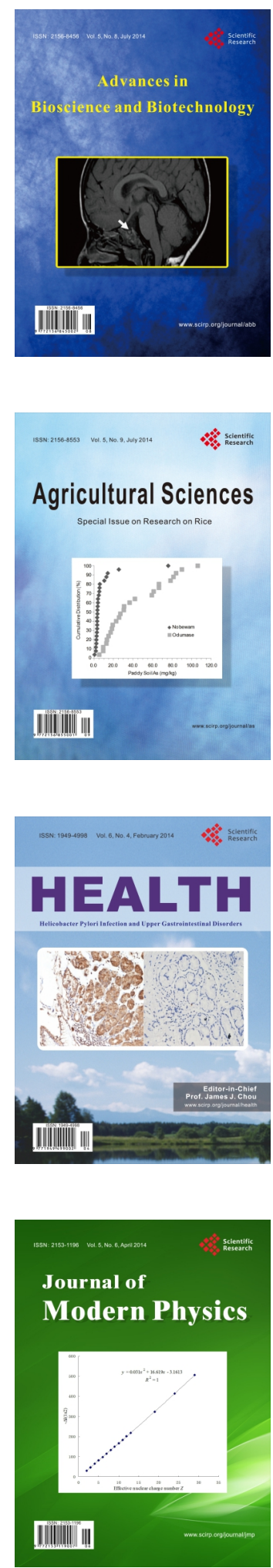
